\title{
THE SERRE PROBLEM ON CERTAIN BOUNDED DOMAINS *
}

\author{
BO-YONG CHEN ${ }^{\dagger}$ AND JINHAO ZHANG ${ }^{\ddagger}$
}

Dedicated to Professor Siu's 60th birthday

\begin{abstract}
We give some new examples for which the Serre problem is solvable by using invariant pseudodistances.

1. Introduction. In 1953, Serre [14] raised the problem whether a holomorphic fiber bundle $\pi: E \rightarrow B$ with a Stein base $B$ and a Stein fiber $F$ is Stein. The answer is positive in the case of 0 -dimensional fibers [19] and 1-dimensional fibers (cf. [7], [11], [15]). However, in high dimensional case, there are counterexamples (cf. [2], [17]). There are still some positive examples. Stehle [18] solved the problem for hyperconvex Stein manifolds. Diederich-Fornaess [15] showed that any bounded $C^{2}$ pseudoconvex domains in $\mathbf{C}^{n}$ is hyperconvex, therefore, the Serre conjecture is true in this case. Siu [16] proved the case when the fiber is a bounded pseudoconvex domain in $\mathbf{C}^{n}$ with zero first Betti number. The purpose of this note is to show
\end{abstract}

THEOREM 1. The answer to the Serre problem is positive if the fiber is either of the following:

(i) a bounded domain $\Omega$ in $\mathbf{C}^{n}$ which has a psh exhaustion function such that

$$
\psi \leq c \log \log 1 / \delta_{\Omega},
$$

where $\delta_{\Omega}$ denotes the Euclidean boundary distance;

(ii) a Stein domain of the form $\Omega=\tilde{\Omega} \backslash S$, where $\tilde{\Omega}$ is a bounded domain in $\mathbf{C}^{n}$ which has a continuous bounded psh exhaustion function $\rho$ with $-\rho \leq c \delta_{\Omega}^{\gamma}$ for suitable $c, \gamma>0$, and $S$ is a closed subset of $\tilde{\Omega}$ which is negligible w.r.t. to $L^{2}$ holomorphic functions, i.e., any $L^{2}$ holomorphic function on $\Omega$ extends holomorphically to $\tilde{\Omega}$.

REMARK. a) We will show that any bounded hyperconvex domain together with some non-hyperconvex examples satisfy condition (i).

b) According to [3], any bounded $C^{2}$ pseudoconvex domain has a bounded psh exhaustion function $\rho=-(-r)^{\alpha}$ where $\alpha>0$ and $r$ is a defining function. On the other hand, there are obviously various examples whose boundary is not $C^{2}$, for example, the egg domain defined by $\left\{z \in \mathbf{C}^{n}:\left|z_{1}\right|^{\alpha_{1}}+\cdots+\left|z_{n}\right|^{\alpha_{n}}<1\right\}$ where all $\alpha_{i}>0$.

Acknowledgement. Part of the work was done during the first named author's stay at Graduate School of Mathematics, Nagoya University. He would like to thank the institute, in particular, Professor T. Ohsawa for the hospitality. We also thank W. Zwonek for informing Herbort's example which satisfies the condition of Theorem 1 , and the referee for valuable comments.

\footnotetext{
*Received January 16, 2003; accepted for publication September 1, 2003.

†Department of Applied Mathematics, Tongji University, Shanghai 200092, P. R. China. Current Address: Graduate School of Mathematics, Nagoya University, Chikusa-ku, Nagoya 464-8602, Japan (by-chen@math.nagoya-u.ac.jp). The author is supported by JSPS of Japan.

$\ddagger$ Department of Mathematics, Fudan University, Shanghai 200433, P. R. China (seminar@fudan.edu.cn). The author is supported by project G1998030600 of China.
} 
2. Proof of Theorem 1. We recall the following criterion:

Theorem 2. (cf. Stehlé [18], improved by Mok [11]) Let $\pi: E \rightarrow B$ be a holomorphic fiber bundle with Stein base and fiber. If there exists a psh, not necessary continuous exhaustion function $\psi$ on the fiber $F$ such that $\psi \circ h-\psi$ is bounded for any $h \in$ Aut $F$, then $E$ is Stein. Here Aut $F$ denotes the automorphism group of $F$.

In Stehlé's original criterion, one needs the hypothesis that $\psi$ is continuous and that the assumption of continuity was removed in Mok [11]. that

Proposition 3. If there exists on $F$ an upper semi-continuous function $\phi$ such

(i) $\phi$ is bounded from below by a psh exhaustion function on $F$;

(ii) $\phi \circ h-\phi$ is bounded above for any $h \in$ Aut $F$,

then the answer to the Serre problem is positive.

Proof. By Theorem 2, it suffices to construct a psh exhaustion function $\psi$ on $F$ such that $\psi \circ h-\psi$ is bounded above for any $h \in$ Aut $F$. We consider the following extremal function:

$$
\psi(z)=\sup \{u(z): u \in P S H(F), u \leq \phi\}
$$

where $P S H(F)$ denotes all psh functions in $F$. We claim that $\psi$ is the desired function. Since there exists an exhaustion function belonging to the above class, it follows that $\psi$ is an exhaustion function on $F$. Since $\phi$ is upper semi-continuous, the upper envelope $\psi^{*}$ of $\psi$ is psh on $F$ and satisfies $\psi^{*} \leq \phi$, which implies $\psi^{*} \leq \psi$. On the other hand, it is obvious that $\psi^{*} \geq \psi$. Hence $\psi=\psi^{*}$, which implies that $\psi$ is a psh function on $F$. By (ii), we have for any $h \in$ Aut $F$

$$
\begin{aligned}
\psi \circ h(z) & =\sup \{u(h(z)): u \in P S H(F), u \leq \phi\} \\
& \leq \sup \{v(z): v \in P S H(F), v \leq \phi \circ h\} \\
& \leq \psi(z)+C_{h} .
\end{aligned}
$$

The proof is complete.

COROLLARY. The answer to the Serre problem is positive if there exists on the fiber a complete invariant pseudodistance relative to a fixed point which is bounded below by a psh exhaustion function.

Proof. Let $d$ denotes the invariant pseudodistance. We can take $\phi(z)=d\left(z_{0}, z\right)$ for some fixed point $z_{0} \in F$. Clearly, for any $h \in$ Aut $F$, one has

$$
\begin{gathered}
\phi \circ h(z)-\phi(z)=d\left(z_{0}, h(z)\right)-d\left(z_{0}, z\right) \\
=d\left(h^{-1}\left(z_{0}\right), z\right)-d\left(z_{0}, z\right) \leq d\left(z_{0}, h^{-1}\left(z_{0}\right)\right) .
\end{gathered}
$$

The result follows immediately from the above proposition.

Let us see some applications. 
a) Siu's distance: Let $D \neq \mathbf{C}$ be a domain in $\mathbf{C}$. Siu [15] constructed an invariant distance on $D$ satisfying

$$
\operatorname{dist}_{S}\left(z_{0}, z\right) \geq \frac{1}{4} \log \frac{\delta_{D}\left(z_{0}\right)}{\delta_{D}(z)} .
$$

Note that the right side is naturally a subharmonic exhaustion function when $D$ is bounded. To define a subharmonic exhaustion function when $D$ is unbounded, one can consider at the same time the domain on the $w$-plane defined by $w=\frac{1}{z-p}$ for some point $p \in \Omega$.

b) Bergman distance: Let $\Omega$ be a bounded domain in $\mathbf{C}^{n}$, and let $K_{\Omega}(z, w)$ be the Bergman kernel and $K_{\Omega}(z)=K_{\Omega}(z, z)$. The Bergman metric is defined by

$$
B_{\Omega}(z ; X)=\left(\sum_{j . k=1}^{n} \frac{\partial^{2} \log K_{\Omega}(z)}{\partial z_{j} \partial \bar{z}_{k}} X_{j} \bar{X}_{k}\right)^{1 / 2}
$$

where $X=\sum_{j=1}^{n} X_{j} \partial / \partial z_{j} \in T^{1,0}\left(\mathbf{C}^{n}\right)$. The related distance is called the Bergman distance. We denote by dist $_{B}$.

Diederich-Ohsawa [4] showed that the Bergman distance satisfies the following estimate for bounded $C^{2}$ pseudoconvex domains

$$
\operatorname{dist}_{B}\left(z_{0}, z\right) \geq C \log \log 1 / \delta_{D}(z)
$$

On the other hand, there exists on $\Omega$ (cf. [9]) a negative psh function $\rho$ satisfying

$$
-\frac{A}{\log 1 / \delta_{\Omega}(z)} \leq \rho(z) \leq-\frac{B}{\log 1 / \delta_{\Omega}(z)}
$$

for suitable positive constants $A, B$. This implies in particular

$$
-\log (-\rho(z)) \leq C^{\prime} \log \log 1 / \delta_{\Omega}(z) .
$$

Note that the left side is also a psh exhaustion function.

c) Kähler-Einstein metric (proof of of Theorem 1): A Kähler-Einstein metric on a complex manifold is a Kähler metric for which the Ricci tensor coincides up to multiplication by a real constant with the metric tensor. Thanks to Mok-Yau [12], such a metric exists on any bounded pseudoconvex domain in $\mathbf{C}^{n}$. Moreover, it is complete, biholomorphically invariant, and the Kähler-Einstein distance dist $_{K E}$ satisfies

$$
\operatorname{dist}_{K E}\left(z_{0}, z\right) \geq C \log \log 1 / \delta_{\Omega}(z) .
$$

Hence (i) of the proof of Theorem 1 follows immediately from Proposition 3.

Before proving (ii), let us recall that the pluricomplex Green function of a bounded domain $\Omega$ with a pole at $w$ is defined by

$$
g_{\Omega}(z, w)=\sup \left\{u(z): u<0, u \in P S H(\Omega), \lim _{z \rightarrow w} \sup (u(z)-\log |z-w|)<+\infty\right\}
$$


It is well-known that $g_{\Omega}(\cdot, w)$ is a psh function and $g_{\Omega}(h(z), h(w))=g_{\Omega}(z, w)$ for any $h \in \operatorname{Aut} \Omega$ (cf. [10]). Set

$$
r_{w}(z)=\max \left\{g_{\Omega}(z, w),-1\right\}
$$

Lemma 4. Let $w, w^{\prime} \in \Omega$. There exist positive constants $C_{1}, C_{2}$ depending only on $w, w^{\prime}$ such that

$$
C_{1} \leq \frac{r_{w}(z)}{r_{w^{\prime}}(z)} \leq C_{2}
$$

for all $z \in \Omega$.

Proof. Without loss of generality, we assume $w \neq w^{\prime}$. Set

$$
\delta=\frac{1}{2} \min \left\{\left|w-w^{\prime}\right|, \delta_{\Omega}(w), \delta_{\Omega}\left(w^{\prime}\right)\right\}
$$

and

$$
\eta(z)= \begin{cases}g\left(z, w^{\prime}\right) & \text { if }\left|z-w^{\prime}\right|<\delta \\ \max \left\{C_{3} r_{w}(z), g\left(z, w^{\prime}\right)\right\} & \text { if }\left|z-w^{\prime}\right| \geq \delta\end{cases}
$$

where $C_{3}=C_{3}\left(w, w^{\prime}\right)$ is a positive constant which satisfies

$$
C_{3} \sup _{\left\{\left|z-w^{\prime}\right|=\delta\right\}} r_{w}(z) \leq \inf _{\left\{\left|z-w^{\prime}\right|=\delta\right\}} g\left(z, w^{\prime}\right)
$$

because $g_{\Omega}\left(z, w^{\prime}\right) \geq \log \left|z-w^{\prime}\right| / R, z \in \Omega$ and $r_{w}$ is upper semi-continuous. Here $R$ denotes the diameter of $\Omega$ and $0<r<\delta_{\Omega}(w)$. Thus $\eta$ is a well-defined negative psh function with a pole at $w^{\prime}$. Hence $g_{\Omega}\left(z, w^{\prime}\right) \geq C_{3} r_{w}(z)$ for $\left|z-w^{\prime}\right| \geq \delta$. It follows that the inequality $r_{w}(z) \leq C_{1} r_{w^{\prime}}(z)$ holds on $\Omega$ for suitable constant $C_{1}>0$. The opposite inequality can be obtained in a similar way.

LEMMA 5. Let $\tilde{\Omega}$ be a bounded domain in $\mathbf{C}^{n}$ such that there exists bounded psh exhaustion function $\rho$ satisfying $-\rho(z) \leq c \delta_{\tilde{\Omega}}^{\gamma}(z)$ for suitable constants $c, \gamma>0$. Suppose $\Omega \subset \tilde{\Omega}$. Then for any $z_{0} \in \Omega$, there is a constant $C_{4}$ such that

$$
-r_{z_{0}}(z) \leq C_{4} \delta_{\tilde{\Omega}}^{\gamma}(z), \forall z \in \Omega .
$$

Proof. Let $R$ denote the diameter of $\Omega$. Similar as above, we set

$$
\eta^{\prime}(z)= \begin{cases}\log \left|z-z_{0}\right| / R & \text { if }\left|z-z_{0}\right|<\delta_{\Omega}\left(z_{0}\right) / 2 \\ \max \left\{\log \left|z-z_{0}\right| / R, C_{5} \rho(z)\right\} & \text { if }\left|z-z_{0}\right| \geq \delta_{\Omega}\left(z_{0}\right) / 2\end{cases}
$$

where $C_{5}$ satisfies

$$
C_{5} \sup _{\left\{\left|z-w^{\prime}\right|=\delta_{\Omega}\left(z_{0}\right) / 2\right\}} \rho(z) \leq \inf _{\left\{\left|z-w^{\prime}\right|=\delta_{\Omega}\left(z_{0}\right) / 2\right\}} \log \left|z-z_{0}\right| / R .
$$

Therefore,

$$
g_{\Omega}\left(z, z_{0}\right) \geq C_{5} \rho(z) \geq c C_{5} \delta_{\tilde{\Omega}}^{\gamma}(z)
$$


for $\left|z-z_{0}\right| \geq \delta_{\Omega}(z) / 2$. On the other hand, we note that $r_{z_{0}} \geq-1$ and $\delta_{\tilde{\Omega}}$ has a uniformly positive lower bound on $\left\{z \in \Omega:\left|z-z_{0}\right| \leq \delta_{\Omega}\left(z_{0}\right) / 2\right\}$ because of the continuity of $\delta_{\tilde{\Omega}}$. Thus the desired inequality follows.

Proof of (ii) of Theorem 1. Set $\psi=-\log \left(-r_{z_{0}}\right)$ for some fixed point $z_{0}$. Clearly, it is psh. Since $r_{z_{0}}(h(z))=r_{h^{-1}\left(z_{0}\right)}(z)$ for any $h \in$ Aut $\Omega$, it follows from Lemma 4 that $\psi \circ h-\psi$ is bounded above; By Lemma 5 , we also have $\psi(z) \geq C \log 1 / \delta_{\tilde{\Omega}}(z), \forall z \in \Omega$. Since $\Omega$ is Stein, according to Mok-Yau [12], if one writes the volume form of the Kähler-Einstein metric as

$$
V_{K E}(z)(i / 2)^{n} d z_{1} \wedge d \bar{z}_{1} \wedge \cdots \wedge d z_{n} \wedge d \bar{z}_{n}
$$

then

$$
\begin{aligned}
& V_{K E}(z)=V_{K E}(h(z))\left|\operatorname{det} h^{\prime}(z)\right|^{2}, \forall h \in \text { Aut } \Omega \\
& V_{K E}(z) \geq \frac{C}{\delta_{\Omega}^{2}(z)\left(\log \delta_{\Omega}(z)\right)^{2}} .
\end{aligned}
$$

By the well-known translation formula of the Bergman kernel function, the ratio $V_{K E} / K_{\Omega}$ is a function which is invariant under Aut $\Omega$. Since $S$ is negligible w.r.t. $L^{2}$ holomorphic functions, we have $K_{\Omega}(z)=K_{\tilde{\Omega}}(z)$ for any $z \in \Omega$. By (1), the function $\phi=\log V_{K E} / K_{\Omega}+N \psi$ satisfies the conditions of Proposition 3 provided the constant $N$ large enough, since

$$
K_{\tilde{\Omega}}(z) \leq K_{B\left(z, \delta_{\tilde{\Omega}}(z)\right)}(z) \leq C_{1}^{\prime} \delta_{\tilde{\Omega}}^{-2 n}(z)
$$

Here $B(p, r) \subset \Omega$ denotes the Euclidean ball with centre $p$ and radius $r$ and $C_{1}^{\prime}>0$ is a constant depending only on $n$.

The class of domains in (i) is quite large since we have the following

Proposition 6. Let $\Omega$ be a bounded hyperconvex domain in $\mathbf{C}^{n}$. Then there exists a continuous psh exhaustion function $\psi$ on $\Omega$ such that

$$
\psi(z) \leq C \log \log 1 / \delta_{\Omega}(z) .
$$

Proof. We proceed the proof with the help of the Bergman kernel function $K_{\Omega}(z)$. Take a cut-off function $\chi: \mathbf{R} \rightarrow[0,1]$ such that $\left.\chi\right|_{(-\infty,-2 \log 2]}=1$ and $\left.\chi\right|_{[-\log 2,+\infty)}=$ 0 . Set

$$
\varphi_{z}=2 n g_{\Omega}(\cdot, z)-\log \left(-g_{\Omega}(\cdot, z)+1\right) .
$$

By a standard limiting procedure, we can solve, according to Lemma 4.4 .1 in [8], the equation

$$
\bar{\partial} u=\bar{\partial} \chi\left(-\log \left(-g_{\Omega}(\cdot, z)+1\right)\right)
$$

in the weak sense together with the estimate

$$
\begin{aligned}
\int_{\Omega}|u|^{2} e^{-\varphi_{z}} d V & \leq \int_{\Omega}\left|\bar{\partial} \chi\left(-\log \left(-g_{\Omega}(\cdot, z)+1\right)\right)\right|_{\sqrt{-1} \partial \bar{\partial} \varphi_{z}}^{2} e^{-\varphi_{z}} d V \\
& \leq C_{2}^{\prime} \operatorname{vol}\left(\left\{g_{\Omega}(\cdot, z)<-1\right\}\right)
\end{aligned}
$$


because

$$
\sqrt{-1} \partial \bar{\partial} \varphi_{z} \geq \frac{\sqrt{-1} g_{\Omega}(\cdot, z) \bar{\partial} g_{\Omega}(\cdot, z)}{\left(-g_{\Omega}(\cdot, z)+1\right)^{2}}
$$

Here $|\cdot|_{\partial \bar{\partial} \varphi_{z}}$ denotes the pointwise norm with respect to the (singular) metric $\partial \bar{\partial} \varphi_{z}$ and $C_{2}^{\prime}$ depends only on $n$ and the choice of $\chi$. Set

$$
f=\chi\left(-\log \left(-g_{\Omega}(\cdot, z)+1\right)\right)-u .
$$

Clearly, $f$ is a holomorphic function on $\Omega$ which satisfies $f(z)=1$ and

$$
\begin{aligned}
\int_{\Omega}|f|^{2} d V & \leq 2 \int_{\Omega}\left|\chi\left(-\log \left(-g_{\Omega}(\cdot, z)+1\right)\right)\right|^{2} d V+2 \int_{\Omega}|u|^{2} d V \\
& \leq C_{3}^{\prime} \operatorname{vol}\left(\left\{g_{\Omega}(\cdot, z)<-1\right\}\right)
\end{aligned}
$$

since $\varphi_{z}<0$ and $\varphi_{z} \leq 2 n \log |\cdot-z|+O(1)$. It follows that

$$
K_{\Omega}(z) \geq\left(C_{3}^{\prime} \operatorname{vol}\left(\left\{g_{\Omega}(\cdot, z)<-1\right\}\right)\right)^{-1} .
$$

In [1], Blocki-Pflug proved that there exists a bounded continuous psh exhaustion function $\rho$ on $\Omega$ such that

$$
\int_{\Omega}\left(-g_{\Omega}(\cdot, z)\right)^{n} d V \leq n !(2 \pi)^{n}\|\rho\|_{L^{\infty}(D)}^{n-1}|\rho(z)|
$$

which implies

$$
\operatorname{vol}\left(\left\{g_{\Omega}(\cdot, z)<-1\right\}\right) \leq C_{4}^{\prime}|\rho(z)|
$$

By (2)-(4), we obtain

$$
-\rho \geq C_{5} \delta_{\Omega}^{2 n}
$$

To complete the proof, we only need to set $\psi=\log (1-\log (-\rho)$ ) (without loss of generality, we may assume $-\rho<1$ on $\Omega$ ).

We have also some Non-hyperconvex examples:

1) Consider the Hartogs domain defined as follows

$$
\Omega=\left\{(z, w) \in D \times \mathbf{C}^{m}:|w|<\exp (-\exp \varphi(z))\right\}
$$

where $D$ is a bounded pseudoconvex domain in $\mathbf{C}^{n}$ and $\varphi$ is an continuous psh exhaustion function of $D$. Set

$$
\psi(z, w)=\max \{\varphi(z), \log (1-\log (1-|w| \exp \exp \varphi(z)))\} .
$$

Clearly, $\psi$ is a psh exhaustion function of $\Omega$. Note that

$$
\delta_{\Omega}((z, w)) \leq \exp (-\exp \varphi(z))-|w|
$$

which implies

$$
\varphi(z) \leq \log \log 1 / \delta_{\Omega}((z, w))
$$


We also have

$$
\begin{aligned}
1-|w| \exp \exp \varphi(z) & =\exp \exp \varphi(z)(\exp (-\exp \varphi(z))-|w|) \\
& \geq \exp (-\exp \varphi(z))-|w| \\
& \geq \delta_{\Omega}((z, w))
\end{aligned}
$$

It follows that

$$
\psi(z, w) \leq C \log \log 1 / \delta_{\Omega}((z, w))
$$

It is well known that $\Omega$ is hyperconvex iff $D$ is hyperconvex. Hence we can obtain various non-hyperconvex examples.

2) Herbort's example (cf. [6]):

$$
\Omega=\left\{\left(z_{1}, z_{2}\right) \in \mathbf{C}^{2}: z_{1} \in \Delta^{*},\left|z_{2}\right|^{2} e^{1 /\left|z_{1}\right|^{2}}<1\right\}
$$

where $\Delta^{*}$ denotes the punctured unit disk. By a similar argument as above, one can show that

$$
\psi(z)=\max \left\{-\log \left|z_{1}\right|, \log \left(1-\log \left(1-\left|z_{2}\right|^{2} e^{1 /\left|z_{1}\right|^{2}}\right)\right)\right\}
$$

satisfies the condition (i) of Theorem 2 .

REMARK. If $\Omega_{1}, \Omega_{2}$ satisfy condition (i) of Theorem 2 , then $\Omega=\Omega_{1} \times \Omega_{2}$ also satisfies this condition: it suffices to take $\psi\left(z^{\prime}, z^{\prime \prime}\right)=\max \left\{\psi_{1}\left(z^{\prime}\right), \psi_{2}\left(z^{\prime \prime}\right)\right\}$ for the slow growth psh exhaustion functions $\psi_{j}$ relative to $\Omega_{j}, j=1,2$.

d) Kobayashi pseudodistance: Let $M$ be a complex manifold and let $\Delta$ denote the unit disk in $\mathbf{C}$. The Kobayashi-Royden pseudometric is defined by

$$
F_{K R}(z ; X):=\inf \left\{|a|^{-1}: \exists f: \Delta \rightarrow M \text { holomorphic with } f(0)=z, f^{\prime}(0)=a X\right\} .
$$

The related pseudodistance is called the Kobayashi pseudodistance which is denoted by $d_{i s t}$.

According to Wu's theorem, any complete simply-connected Kähler manifold of nonpositive sectional curvature is Stein, namely, $\log \left(1+\rho^{2}\right)$ is a strictly psh exhaustion function. Here $\rho$ denotes the distance function relative to some fixed point of $M$. If furthermore, the holomorphic sectional curvature is bounded from above by $-\frac{A}{1+\rho^{2}}$, then $M$ is complete hyperbolic [5], moreover, the Kobayashi distance satisfies

$$
\operatorname{dist}_{K}\left(z_{0}, z\right) \geq C \log \left(1+\rho^{2}(z)\right) .
$$

Thus we obtain the following

THEOREM 7. The answer to the Serre problem is affirmative if the fiber is a complete simply-connected Kähler manifold of nonpositive sectional curvature such that the holomorphic sectional curvature is bounded above by $-\frac{A}{1+\rho^{2}}$. 


\section{REFERENCES}

[1] Z. Blocki And P. Pflug, Hyperconvexity and Bergman completeness, Nagoya Math. J., 151 (1998), pp. 221-225.

[2] G. Coeuré And J. J. Loeb, A counterexample to the Serre problem with a bounded domain in $\mathbf{C}^{2}$ as fiber, Ann. of Math., 122 (1985), pp. 329-334.

[3] K. Diederich and J. E. Fornaess, Pseudoconvex domains: Bounded strictly plurisubharmonic exhaustion functions, Invent. Math., 39 (1977), pp. 129-141.

[4] K. Diederich And T. Ohsawa, An estimate for the Bergman distance on pseudoconvex domains, Ann. of Math., 141 (1995), pp. 181-190.

[5] R. E. GREene AND H. WU, Function theory on manifolds which possess a pole, Lecture Notes in Mathematics, 699, Springer-Verlag 1979.

[6] G. Herbort, The Bergman metric on hyperconvex domains, Math. Z., 232 (1999), pp. $183-196$.

[7] A. Hirschowitz, Domains de Stein et fonctions holomorphes bornées, Math. Ann., 213 (1975), pp. 185-193.

[8] L. Hörmander, An introduction to Complex Analysis in Several Variables, North Holland 1990.

[9] N. Kerzman AND J. P. Rosay, Fonctions plurisousharmonique d'exhaustion bornées et domaines taut, Math. Ann., 257 (1981), pp. 171-184.

[10] M. KLIMEK, Extremal plruisubharmonic functions and invariant pseudodistances, Bull. Soc. Math. France, 113 (1985), pp. 123-142.

[11] N. MoK, The Serre problem on Riemann Surfaces, Math. Ann., 258 (1981), pp. 145-168.

[12] N. MOK AND S. T. YAU, Completeness of the Kähler-Einstein metric on bounded domains and characterization of domains of holomorphy by curvature conditions, Proc. Symp. Pure Math., 39 (1983), pp. 41-60.

[13] P. Pflug, Quadratintegrable holomorphe Functionen und die Serre-Vermutung, Math. Ann., 216 (1975), pp. 285-288.

[14] J. P. SERre, Quelques problèmes globaux relatifs aux variétés de Stein, Colloque sur les Fonctions de Plusieurs Variables Complexes, Bruxelles 1953, pp. 57-68.

[15] Y. T. SiU, All palne domains are Banach-Stein, Manuscr. Math., 14 (1974), pp. 101-105.

[16] - Holomorphic fiber bundles whose fibers are bounded Stein domains with zero first Betti number, Math. Ann., 219 (1976), pp. 171-192.

[17] H. SKodA, Fibrés holomorphes à base et à fibre de Stein, Invent. Math., 43 (1977), pp. 97-107.

[18] J. L. Stehlé, Fonctions plurisousharmoniques et convexité holomorphe de certain fibrés analytiques, Lecture Notes in Math. Séminaire P. Lelong, Springer Verlag, 474 (1973/1974), pp. 155-179.

[19] K. STEIN, Uberlagerungen holomorph-vollstandiger komplexer Raume, Arch. Math., 7 (1956), pp. 354-361. 